\title{
Optimization of the Norm of a Vector-Valued DC Function and Applications ${ }^{1}$
}

\author{
R. Blanquero and E. CARRIZOSA ${ }^{3}$ \\ Communicated by P. Pardalos
}

\begin{abstract}
In this paper, we show that a DC representation can be obtained explicitly for the composition of a gauge with a DC mapping, so that the optimization of certain functions involving terms of this kind can be made by using standard DC optimization techniques. Applications to facility location theory and multiple-criteria decision making are presented.
\end{abstract}

Key Words. Global optimization, difference of convex functions, location theory, multiple-criteria decision making.

\section{Problem Formulation}

DC functions (functions $\psi$ that can be written as the difference of two convex functions $\psi^{+}, \psi^{-}$) constitute a wide class of functions that plays an important role within the field of global optimization (R efs. 1-3). A mong other properties, the class of DC functions is closed under certain operations. F or instance, Proposition 4 in R ef. 3 asserts that the composition of $D C$ functions is also a DC function. Since the proof is based on the fact that any locally DC function on a convex set is also DC (R ef. 4), it is a nonconstructive proof; i.e., it does not provide a $D C$ decomposition for $\psi=\gamma \circ f$, even when DC decompositions for $f$ and $\gamma$ are known. From an optimization point of view, this is an important drawback, since most powerful DC optimization techniques, such as branch-and-bound methods (R ef. 1), need a DC representation of the function under study. For this reason, specific methods for obtaining $D C$ decompositions have been proposed for particular functions $\gamma$; see for example Propositions 3.5-3.7 in R ef. 5.

\footnotetext{
${ }^{1}$ This research was partially supported by G rant PB 96-1416-C O2-02, D GES, M adrid, Spain. ${ }^{2}$ Professor, F acultad de M atemáticas, U niversidad de Sevilla, Sevilla, Spain.

${ }^{3}$ Professor, F acultad de M atemáticas, U niversidad de Sevilla, Sevilla, Spain.
} 
In this paper, we consider the particular case of compositions of DC functions in which the outer function $\gamma$ is a gauge, that is, a convex function $\gamma: \mathbb{R}^{m} \rightarrow \mathbb{R}$, defined as

$$
\gamma(x)=\inf \{t>0: x \in t B\}, \quad x \in \mathbb{R}^{m},
$$

where $B$ is a convex set, the interior of which contains the origin (R efs. 6-7).

By Theorem 14.5 in R ef. 7, every gauge $\gamma$ can be written as

$$
\gamma(x)=\max \left\{\langle u, x\rangle: u \in B^{0}\right\}, \quad x \in \mathbb{R}^{m},
$$

where $\langle\cdot, \cdot\rangle$ denotes the usual scalar product and $B^{0}$ is the polar set of $B$, that is

$$
B^{0}=\{v:\langle v, x\rangle \leq 1, \forall x \in B\} .
$$

$\mathrm{U}$ sing this property, the proof of Proposition 4 in R ef. 3 can be rewritten for this particular case, yielding a global DC decomposition for $\gamma \circ f$, as shown below.

Proposition 1.1. Let $\Omega \subset \mathbb{R}^{n}$ be a convex set. Let $\gamma: \mathbb{R}^{m} \rightarrow \mathbb{R}$ be a gauge in $\mathbb{R}^{m}$ with unit ball $B$, let

$$
f=\left(f_{1}, \ldots, f_{m}\right): \Omega \rightarrow \mathbb{R}^{m}
$$

be a DC vector-valued function, with known DC decomposition,

$$
f_{i}=f_{i}^{+}-f_{i}^{-},
$$

with $f_{i}^{+}$and $f_{i}^{-}$convex. For any $i=1, \ldots, m$, let

$$
M_{i} \geq \max \left\{\gamma\left(e_{i}\right), \gamma\left(-e_{i}\right)\right\},
$$

where $e_{i}$ is the $i$ th unit vector of $\mathbb{R}^{m}$. Then, $\gamma \circ f: \Omega \rightarrow \mathbb{R}$ is a DC function, and a $\mathrm{DC}$ decomposition for it is given by

$$
\gamma \circ f=g-h,
$$

with

$$
g=\gamma \circ f+\sum_{i=1}^{m} M_{i}\left(f_{i}^{+}+f_{i}^{-}\right), \quad h=\sum_{i=1}^{m} M_{i}\left(f_{i}^{+}+f_{i}^{-}\right) .
$$

Proof. First, observe that a finite

$$
M_{i} \geq \max \left\{\gamma\left(e_{i}\right), \gamma\left(-e_{i}\right)\right\}
$$

can be chosen, since the origin is an interior point of $B$ and, therefore, the polar set $B^{0}$ is bounded. F rom (2), the gauge $\gamma$ can be globally represented as a pointwise maximum of the affine functions $\varphi_{u}$, 


$$
\gamma(y)=\max _{u \in B^{0}} \varphi_{u}(y), \quad \forall y \in \mathbb{R}^{m}
$$

where

$$
\varphi_{u}(y)=\langle u, y\rangle .
$$

Then,

$$
\begin{aligned}
\varphi_{u}\left(f_{1}, \ldots, f_{m}\right)= & \sum_{i=1}^{m} u_{i} f_{i}^{+}-\sum_{i=1}^{m} u_{i} f_{i}^{-} \\
= & \sum_{i=1}^{m}\left(M_{i}+u_{i}\right) f_{i}^{+}+\sum_{i=1}^{m}\left(M_{i}-u_{i}\right) f_{i}^{-} \\
& -\sum_{i=1}^{m} M_{i}\left(f_{i}^{+}+f_{i}^{-}\right)
\end{aligned}
$$

Since

$$
M_{i} \geq \gamma\left(e_{i}\right)=\max _{u \in B^{0}} u_{i}
$$

and

$$
M_{i} \geq \gamma\left(-e_{i}\right)=\max _{u \in B^{0}}-u_{i},
$$

it follows that $f$ can be written as the difference of two convex functions, namely,

$$
\varphi_{u} \circ f=p_{u}-q_{1}
$$

where

$$
\begin{aligned}
& p_{u}=\sum_{i=1}^{m}\left(M_{i}+u_{i}\right) f_{i}^{+}+\sum_{i=1}^{m}\left(M_{i}-u_{i}\right) f_{i}^{-}, \\
& q=\sum_{i=1}^{m} M_{i}\left(f_{i}^{+}+f_{i}^{-}\right) .
\end{aligned}
$$

Then,

$$
\begin{aligned}
\gamma \circ f & =\max _{u \in B^{0}} \varphi_{u}\left(f_{1}, \ldots, f_{m}\right) \\
& =\max _{u \in B^{0}}\left(p_{u}-q\right) \\
& =\left(\max _{u \in B^{0}} p_{u}\right)-q \\
& =(\gamma \circ f+q)-q
\end{aligned}
$$

and the result holds. 
This provides immediately DC decompositions for important classes of DC vector-valued functions. In particular, if the gauge $\gamma$ is an $L_{p}$-norm, it can be taken

$$
M_{i}=1, \quad \text { for every } i,
$$

yielding the following corollary.

Corollary 1.1. Let $f_{1}, \ldots, f_{m}$ be $\mathrm{DC}$ functions on the convex set $\Omega$, with DC decompositions $f_{i}=f_{i}^{+}-f_{i}^{-}, i=1, \ldots, m$. Then, for any $p$, $1 \leq p \leq \infty,\|f\|_{p}$ is $\mathrm{DC}$ on $\Omega$, a $\mathrm{DC}$ decomposition being given by

$$
\|f\|_{p}=g-h \text {, }
$$

with

$$
g=\|f\|_{p}+\sum_{i=1}^{m}\left(f_{i}^{+}+f_{i}^{-}\right), \quad h=\sum_{i=1}^{m}\left(f_{i}^{+}+f_{i}^{-}\right) .
$$

The result in Proposition 1.1 can be strengthened if further assumptions are made on the gauge $\gamma$ in use. Indeed, the proof above can be rewritten e.g. for the case in which the polar ball $B^{0}$ is contained in the positive orthant.

Proposition 1.2. Let $\Omega \subset \mathbb{R}^{n}$ be a convex set. Let $\gamma: \mathbb{R}^{m} \rightarrow \mathbb{R}$ be a gauge in $\mathbb{R}^{m}$ with unit ball $B$, such that $B^{0} \subset \mathbb{R}^{m}$. L et $f=\left(f_{1}, \ldots, f_{m}\right): \Omega \rightarrow \mathbb{R}^{m}$ be a $\mathrm{DC}$ vector-valued function, with known $\mathrm{DC}$ decomposition, $f_{i}=f_{i}^{+}-f_{i}^{-}$, with $f_{i}^{+}$and $f_{i}^{-}$convex. For any $i=1, \ldots, m$, let $M_{i} \geq \gamma\left(e_{i}\right)$, where $e_{i}$ is the $i$ th unit vector of $\mathbb{R}^{m}$. Then, $\gamma \circ f: \Omega \rightarrow \mathbb{R}$ is a $D C$ function, and a DC decomposition for it is given by

$$
\gamma \circ f=g-h,
$$

with

$$
g=\gamma \circ f+\sum_{i=1}^{m} M_{i} f_{i}^{-}, \quad h=\sum_{i=1}^{m} M_{i} f_{i}^{-} .
$$

A s a nontrivial application, let us obtain a DC decomposition for the $k$ th function: given $f=\left(f_{1}, \ldots, f_{m}\right)$, let $\left(f_{(1)}(x), \ldots, f_{(m)}(x)\right)$ be the arrangement of $\left(f_{1}(x), \ldots, f_{m}(x)\right)$ with

$$
f_{(1)}(x) \geq f_{(2)}(x) \geq \cdots \geq f_{(m)}(x) .
$$

Then, we have the following proposition. 
Proposition 1.3. Let $\Omega \subset \mathbb{R}^{n}$ be a convex set. Let $f=$ $\left(f_{1}, \ldots, f_{m}\right): \Omega \rightarrow \mathbb{R}^{m}$ be a $\mathrm{DC}$ vector-valued function, with known $\mathrm{DC}$ decomposition $f_{i}=f_{i}^{+}-f_{i}^{-}$, with $f_{i}^{+}$and $f_{i}^{-}$convex. Let

$$
h=\sum_{i=1}^{m} f_{i}^{-}
$$

and, for any $k=1, \ldots, m$, let

$$
g_{k}=f_{(1)}+f_{(2)}+\cdots f_{(k)}+\sum_{i=1}^{m} f_{i}^{-} .
$$

Then, a $\mathrm{DC}$ decomposition for $f_{(1)}+f_{(2)}+\cdots+f_{(k)}$ is given by

$$
f_{(1)}+f_{(2)}+\cdots+f_{(k)}=g_{k}-h .
$$

In particular, a DC decomposition for $f_{(k)}$ is given by

$$
f_{(k)}=\left(g_{k}+h\right)-\left(g_{k-1}+h\right) \text {. }
$$

Proof. The decomposition (5) follows from the fact that

$$
f_{(1)}+\cdots+f_{(k)}=\max \left\{\langle f, u\rangle: \sum_{i=1}^{m} u_{i}=k, 0 \leq u_{i} \leq 1, \forall i\right\} .
$$

Thus, we can use Proposition 1.3 for $B^{0}$ given by

$$
B^{0}=\left\{u \in \mathbb{R}^{m}: \sum_{i=1}^{m} u_{i}=k, 0 \leq u_{i} \leq 1, \forall i\right\} .
$$

The decomposition (6) follows from (5) and the fact that

$$
f_{(k)}=\left[f_{(1)}+\cdots+f_{(k)}\right]-\left[f_{(1)}+\cdots+f_{(k-1)}\right] .
$$

\section{Applications}

In this section, we analyze applications to the fields of location theory and multiple-criteria decision making that justify the interest of Proposition 1.1.

2.1. Facility Location. As an application of Proposition 1.1 to facility location theory, we consider the Fermat-Weber problem with forbidden regions and mixed gauges. The aim is to find a location outside int $(S)$, the interior of a region $S$, for a new facility in the plane in such a way that the weighted sum of the distances between the facility and the demand points 
(fixed facilities) is minimized; that is, we have to solve the optimization problem

$$
\min \left\{\sum_{i=1}^{n} \gamma_{i}\left(x-a_{i}\right): x \in \mathbb{R}^{2} \backslash \operatorname{int}(S)\right\},
$$

were $\gamma_{i}$ is a gauge measuring the distance from any point in the plane to $a_{i}$, $x$ is the unknown location of the new facility, $a_{i}$ is the location of the $i$ th demand point, and $S$ is a subset of $\mathbb{R}^{2}$ that can be represented as the union of $m$ pairwise disjoint connected (not necessarily convex) sets,

$$
S=\bigcup_{j=1}^{m} S_{j}
$$

The usual strategy for finding an optimal solution of (7) consists of first solving the unconstrained problem, and then checking if any element in the set $X^{*}$ of optimal solutions is a feasible point for problem (7). If this is the case, then we have an optimal location for the restricted problem. In other cases, the convexity of the objective function allows us to ensure the existence of an optimal solution belonging to $\operatorname{bd}(S)$, the boundary of $S$; thus, we just need to solve, for $j=1, \ldots, m$,

$$
\min \left\{\sum_{i=1}^{n} \gamma_{i}\left(x-a_{i}\right): x \in \mathrm{bd}\left(S_{j}\right)\right\} .
$$

M ost papers in the literature do not address the problem in its full generality, since they impose strong assumptions on the gauge (assumed to be the $L_{1}$ norm or $L_{2}$ norm) and the forbidden region, which is considered to have an extremely simple form (a polyhedron or a circle); see R efs. 810.

Suppose that a parametric description for $\mathrm{bd}\left(S_{j}\right)$ is known; i.e., we have a function $w_{j}:[0,1] \mapsto \mathbb{R}^{2}$ parametrizing bd $\left(S_{j}\right)$. Then, (8) can be written as

$$
\min _{0 \leq t \leq 1} \sum_{i=1}^{n} \gamma_{i}\left(w_{j}(t)-a_{i}\right)
$$

which is a one-dimensional optimization problem whose objective function will be multimodal in general, so global optimization techniques must be used for its solution.

In the particular case in which $w_{j}$ is a DC function with a known DC decomposition, Proposition 1.1 provides a $D C$ representation for the objective of (9), so the optimal solution can be obtained by solving a DC univariate problem. Several results given in the literature enable us to obtain such D C decomposition, either directly or by using simpler functions with known representation ( $\mathrm{R}$ efs. $1,3,5,11,12$ ). F or instance, if $w_{j}$ is twice continuously 
differentiable and $K \geq 0$ is a bound for the second derivative, a DC decomposition for $w_{j}$ is given by

$$
w_{j}(t)=\left[w_{j}(t)+(1 / 2) K t^{2}\right]-(1 / 2) K t^{2} .
$$

Example 2.1. A s illustration of this technique, we have considered the following Weber problem with a forbidden region:

$$
\begin{aligned}
& \min \sum_{i=1}^{3} \omega_{i}\left\|x-a_{i}\right\|_{2}, \\
& \text { s.t. } \quad x \in \mathbb{R}^{2} \backslash \operatorname{int}(S),
\end{aligned}
$$

where

$$
\begin{aligned}
& a_{1}=(0,3), \quad a_{2}=(-2,4), \quad a_{3}=(4,-2), \\
& w_{1}=2, \quad w_{2}=3, \quad w_{3}=2,
\end{aligned}
$$

and $S$ is the region enclosed by the curve $R$, a parametric description of which is given by

$$
R=\{(u(t), v(t)), t \in[0,1]\},
$$

with

$$
\begin{aligned}
& u(t)=5 \cos (8 \pi t) \cos (2 \pi t), \\
& v(t)=5 \cos (8 \pi t) \sin (2 \pi t) .
\end{aligned}
$$

The optimal solution for the unconstrained problem is $a_{1}=(0,3)$, an interior point of $S$ (see Fig. 1), so the optimal solution for the constrained problem will be located at its boundary.

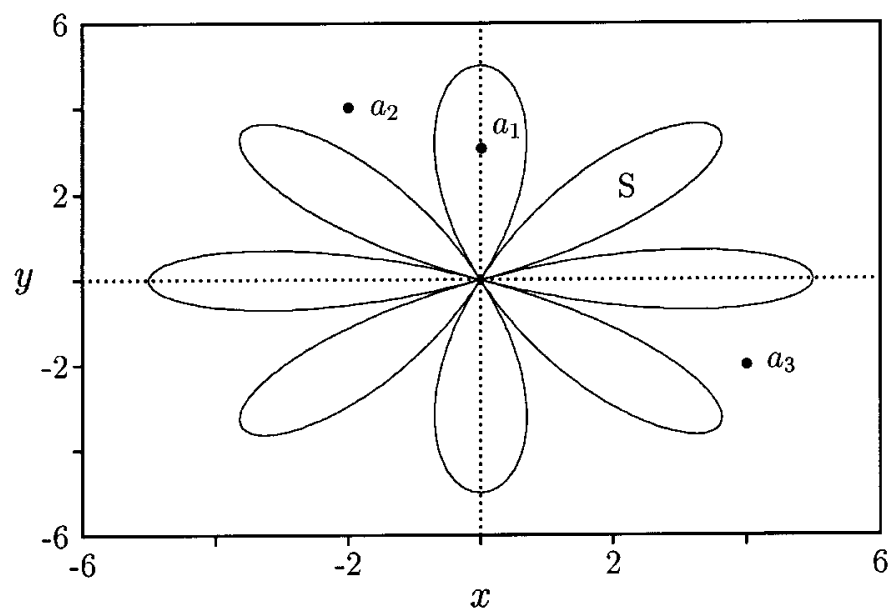

Fig. 1. Forbidden region and demand points, Example 2.1. 
Taking into account that a parametric description for $\mathrm{bd}(S)$ is already given, the optimal solution can be computed by solving

$$
\min _{t \in[0,2 \pi]} F(t):=\sum_{i=1}^{3} \omega_{i}\left\|\left(u(t)-a_{i 1}, v(t)-a_{i 2}\right)\right\|_{2} .
$$

The objective is a multimodal function, as can be seen in Fig. 2 , so the use of global optimization techniques is required.

Since $u$ and $v$ are $C^{2}$ in $[0,1]$, they are $\mathrm{DC}$ functions and (10) provides the $\mathrm{DC}$ decomposition

$$
u(t)=u^{+}(t)-u^{-}(t), \quad v(t)=v^{+}(t)-v^{-}(t),
$$

with

$$
\begin{array}{ll}
u^{+}(t)=5 \cos (8 \pi t) \cos (2 \pi t)+170 \pi^{2} t^{2}, & u^{-}(t)=170 \pi^{2} t^{2}, \\
v^{+}(t)=5 \cos (8 \pi t) \sin (2 \pi t)+170 \pi^{2} t^{2}, & v^{-}(t)=170 \pi^{2} t^{2} .
\end{array}
$$

Hence, by Corollary 1.1, it can be claimed that $[F(t)+h(t)]-h(t)$ is a DC decomposition for $F$, with

$$
\begin{aligned}
h(t) & =7\left[u^{+}(t)+v^{+}(t)+u^{-}(t)+v^{-}(t)\right]-16 \\
& =7\left[5 \cos (8 \pi t) \cos (2 \pi t)+5 \cos (8 \pi t) \sin (2 \pi t)+680 \pi^{2} t^{2}\right]-16 .
\end{aligned}
$$

A $n \epsilon$-optimal solution for the problem above has been obtained by using a covering algorithm ( $R$ ef. 13 ), yielding

$$
t^{*}=0.28527653858 \text {, }
$$

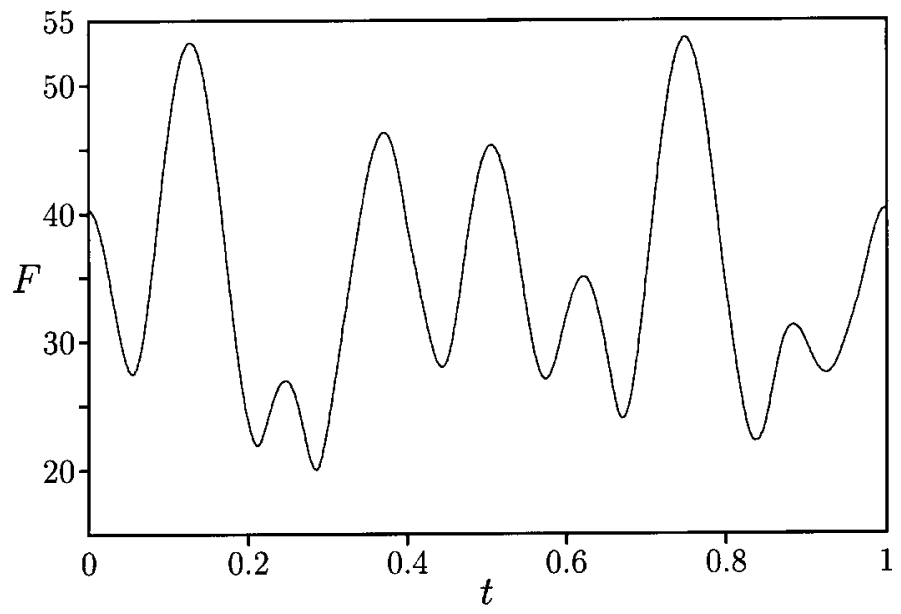

Fig. 2. Objective function over bd(S), Example 2.1. 
which corresponds to the point

$$
x^{*}=(-0.6947487405,3.082955211) .
$$

Whereas it might be the case, as in Example 2.1, that a DC parametrization of $\mathrm{bd}\left(S_{j}\right)$ is already given, in general such parametrization must also be determined. Fortunately, this is an easy task when $S_{j}$ is a convex and compact set. Indeed, assuming without loss of generality that the origin is an interior point of $S_{j}$, we have the following parametric description of its boundary:

$$
\begin{aligned}
\omega_{j}(t)= & {\left[\cos (2 \pi t) / \gamma_{j}(\cos 2 \pi t, \sin 2 \pi t),\right.} \\
& \left.\sin (2 \pi t) / \gamma_{j}(\cos 2 \pi t, \sin 2 \pi t)\right], \quad t \in[0,1],
\end{aligned}
$$

where $\gamma_{j}$ is the gauge with unit ball $S_{j}$. Both $\cos (2 \pi t)$ and $\sin (2 \pi t)$ are DC functions; so, by Proposition 1.1, $\gamma_{j}(\cos 2 \pi t, \sin 2 \pi t)$ is also DC (with a DC decomposition at hand); thus, every component of (11) is the quotient of two DC functions, thus DC.

In order to solve (9) with the $\omega_{j}(t)$ in (11) via e.g. a branch-and-bound method, we will simply need a DC decomposition and a procedure for evaluating the objective function and constructing the subgradients at given points.

In the search of a DC representation for every component of $w$, it suffices to obtain a $D C$ decomposition for the quotient of a convex function and a DC function, since the numerators of (11) can be expressed easily as the difference of two convex functions. In order to achieve this, we need the following lemma.

Lemma 2.1. Let $U$ be a bounded set with $U \subset\left\{(x, y, z) \in \mathbb{R}^{3}: y-z \geq \alpha\right\}$, where $\alpha>0$, and let $u$ : $U \mapsto \mathbb{R}$ be defined as $u(x, y, z)=x \cdot(y-z)^{-1}$. Then, there exist $\rho^{*}, A, B, C \in \mathbb{R}$ such that

$$
\begin{aligned}
& u^{+}(x, y, z)=u(x, y, z)+(1 / 2) \rho^{*}\left(x^{2}+y^{2}+z^{2}\right)+A x+B y+C z, \\
& u^{-}(x, y, z)=(1 / 2) \rho^{*}\left(x^{2}+y^{2}+z^{2}\right)+A x+B y+C z
\end{aligned}
$$

are convex and increasing componentwise functions.

Proof. The Hessian matrix for $u$ is given by

$$
H=\left[1 /(y-z)^{2}\right]\left[\begin{array}{rll}
0 & -1 & 1 \\
-1 & 2 x /(y-z) & -2 x /(y-x) \\
1 & -2 x /(y-z) & 2 x /(y-z)
\end{array}\right]
$$


and its eigenvalues are

$$
\begin{aligned}
& \lambda_{1}=0, \\
& \lambda_{2}=\left[2 x+\sqrt{4 x^{2}+2(y-z)^{2}}\right] /(y-z)^{3}, \\
& \lambda_{3}=\left[2 x-\sqrt{4 x^{2}+2(y-z)^{2}} /(y-z)^{3},\right.
\end{aligned}
$$

from which one derives immediately the following upper bound for the spectral radius of $H$ :

$$
\rho^{*}=\left[2 M_{|x|}+\sqrt{4 M_{|x|}^{2}+2 m_{y-z}^{2}}\right] / m_{y-z}^{3},
$$

where

$$
\begin{aligned}
& M_{|x|} \geq \sup \{|x|:(x, y, z) \in U\}, \\
& 0<m_{y-z} \leq \inf \{y-z:(x, y, z) \in U\} .
\end{aligned}
$$

By the choice of $\rho^{*}$, it follows that the functions

$$
\begin{aligned}
& u_{1}(x, y, z)=u(x, y, z)+(1 / 2) \rho^{*}\left(x^{2}+y^{2}+z^{2}\right), \\
& u_{2}(x, y, z)=(1 / 2) \rho^{*}\left(x^{2}+y^{2}+z^{2}\right)
\end{aligned}
$$

are convex and that $u^{+}$and $u^{-}$are also convex for any $A, B, C$.

In order to make $u^{+}$and $u^{-}$componentwise increasing, we choose $A, B$, $C$ in such a way that the partial derivatives of these functions with respect to $x, y, z$ are nonnegative. This yields the following lower bounds for these constants:

$$
\begin{aligned}
& A \geq-\rho^{*} m_{x}, \\
& B \geq \max \left\{M_{x} / m_{y-z}^{2}-\rho^{*} m_{y},-\rho^{*} m_{y}\right\}, \\
& C \geq \max \left\{-m_{x} / m_{y-z}^{2}-\rho^{*} m_{z},-\rho^{*} m_{z}\right\},
\end{aligned}
$$

with

$$
\begin{aligned}
& m_{x} \leq \inf \{x:(x, y, z) \in U\}, \\
& M_{x} \geq \sup \{x:(x, y, z) \in U\}, \\
& m_{y} \leq \inf \{y:(x, y, z) \in U\}, \\
& m_{z} \leq \inf \{z:(x, y, z) \in U\},
\end{aligned}
$$

and the result holds.

It is now possible to provide a $D C$ decomposition for the quotient of a convex function and a DC function, from which we obtain a $D C$ representation for $\omega_{j}$ in (11). 
Proposition 2.1. Let $T \subset \mathbb{R}^{k}$ be a convex set, and let $f, g, h: T \mapsto \mathbb{R}$ be finite convex functions such that $\inf \{g(t)-h(t): t \in T\}>0$. Then, a DC representation for $f(t) \cdot[g(t)-h(t)]^{-1}$ is given by

$$
f(t) /[g(t)-h(t)]=\{f(t) /[g(t)-h(t)]+n(t)\}-n(t),
$$

where

$$
n(t)=(1 / 2) \rho^{*}\left[f^{2}(t)+g^{2}(t)+h^{2}(t)\right]+A f(t)+B g(t)+C h(t)
$$

and

$$
\begin{aligned}
& \rho^{*}=\left[2 M_{|f|}+\sqrt{4 M_{|f|}^{2}+2 m_{g-h}^{2}}\right] / m_{g-h}^{3}, \\
& A \geq-\rho^{*} m_{f}, \\
& B \geq \max \left\{M_{f} / m_{g-h}^{2}-\rho^{*} m_{g},-\rho^{*} m_{g}\right\}, \\
& C \geq \max \left\{m_{f} / m_{g-h}^{2}-\rho^{*} m_{h},-\rho^{*} m_{h}\right\},
\end{aligned}
$$

and

$$
\begin{aligned}
& m_{f} \leq \inf _{t \in T} f(t), \\
& M_{f} \geq \sup _{t \in T} f(t), \\
& M_{|f|} \geq \sup _{t \in T}|f(t)|, \\
& m_{g} \leq \inf _{t \in T} g(t), \\
& m_{h} \leq \inf _{t \in T} h(t), \\
& 0<m_{g-h} \leq \inf _{t \in T}\{g(t)-h(t)\} .
\end{aligned}
$$

Proof. Consider the convex mapping

$$
F: t \in T \mapsto F(t)=(f(t), g(t), h(t)) \in \mathbb{R}^{3} .
$$

Since $u^{+}$and $u^{-}$in Lemma 2.1 are convex and increasing componentwise, a basic result from convex analysis (R ef. 14) asserts that $u^{+} \circ F$ and $u^{-} \circ F$ are convex functions, providing a $D C$ decomposition for $u \circ F$. The constants involved in the definition of $n(t)$ are directly taken from the proof of L emma 2.1.

Remark 2.1. A lthough the use of this representation can be hard for general functions, it allows us to find a DC decomposition for (11) with no great effort. Finding a $D C$ representation for $\sin (2 \pi t)$ and $\cos (2 \pi t)$ is a 
trivial task and, from here, one obtains immediately the bounds $m_{f}, M_{f}$, $M_{|f|}, m_{g}, m_{h}$. The calculation of bound $m_{g-h}$ may be a little more difficult, since it is related to the optimal value of $\min _{t \in[0,1]} \gamma(\cos 2 \pi t, \sin 2 \pi t)$. This is a DC optimization problem and a DC decomposition for the objective is known from Proposition 1.1, so one can solve it by applying the covering algorithm provided in R ef. 13 or any other DC optimization method. In fact, since one needs only a lower bound for the optimal value, it suffices to perform some iterations of that algorithm in order to obtain it.

Thanks to Proposition 2.1, in order to solve (9) by branch-and-bound methods, we need only a procedure to evaluate $\gamma_{j}$ and find a subgradient of $\gamma_{j}$ at a given point. This is easy when $S_{j}$ is given in the form

$$
S_{j}=\left\{x \in \mathbb{R}^{2}: g_{j}(x) \leq 0\right\},
$$

for some convex function $g_{j}$ with bounded level sets and $g_{j}(0)<0$. Indeed, since by the definition of the gauge, for any nonzero $x$,

$$
\gamma_{j}(x)=\sup \left\{s>0, g_{j}(s x) \leq 0\right\}^{-1},
$$

evaluating $\gamma_{j}(x)$ amounts to solving the nonlinear univariate equation $g(s x)=0$, for which we can use e.g. any line-search method (R ef. 15).

R egarding the subdifferential, a subgradient of $\gamma_{j}$ at a given point can be computed by using the following result.

Proposition 2.2. Let $g: \mathbb{R}^{n} \mapsto \mathbb{R}$ be a convex function such that the set $S=\left\{x \in \mathbb{R}^{n}: g(x) \leq 0\right\}$ is compact and $g(0)<0$. Let $\bar{x} \neq 0$, and let $u \in \partial g(\bar{x} / \gamma(\bar{x}))$, where $\gamma$ is the gauge with unit ball $S$. Then,

$$
[\gamma(\bar{x}) /\langle u, \bar{x}\rangle] u \in \partial \gamma(\bar{x}) .
$$

Proof. Let $\bar{y}=\bar{x} / \gamma(\bar{x})$, thus $g(\bar{y})=0$. Since $u \in \partial g(\bar{y})$, given $x \in S$ one has the following:

$$
0 \geq g(x) \geq g(\bar{y})+\langle u, x-\bar{y}\rangle ;
$$

thus,

$$
\langle u, x\rangle \leq\langle u, \bar{y}\rangle, \quad \text { for each } x \in S .
$$

Since $\bar{y} \in S$, it follows from the previous relation and the definition of dual gauge that

$$
\gamma^{0}(u)=\max _{x \in S}\langle u, x\rangle=\langle u, \bar{y}\rangle
$$

therefore,

$$
\left\langle u / \gamma^{0}(u), \bar{y}\right\rangle=\left[1 / \gamma^{0}(u)\right]\langle u, \bar{y}\rangle=1=\gamma(\bar{y}) .
$$


Then, we conclude that a subgradient of $\gamma$ at $\bar{y}$ is given by

$$
\left[1 / \gamma^{0}(u)\right] u=[\gamma(\bar{x}) /\langle u, \bar{x}\rangle] u,
$$

and taking into account that

$$
\partial \gamma(\bar{y})=\partial \gamma(\bar{x}),
$$

the result follows.

2.2. Multiple-Criteria Decision Making. Compromise programming (R efs. 16-18) provides an effective tool in multiple-criteria decision making (M CD M ). G iven a multiple-objective problem

$$
\max _{x \in X} f(x):=\left(f_{1}(x), \ldots, f_{m}(x)\right),
$$

with $X \subset \mathbb{R}^{n}$, in compromise programming one proceeds as follows: compute the ideal point of the problem

$$
\bar{f}=\left(\bar{f}_{1}, \ldots, \bar{f}_{m}\right),
$$

where

$$
\bar{f}_{i}=\max _{x \in X} f_{i}(x) .
$$

If $\bar{f}$ corresponds to a feasible point (which seldom happens), it will be the optimal solution; in the other case, the best compromise solution is provided, that is, the output closest to the ideal point. The distance between every feasible solution and the ideal point is measured via an $L_{p}$-metrics, $1 \leq p \leq \infty$, namely,

$$
\left\{\sum_{i=1}^{m} w_{i}^{p}\left|\left[\bar{f}_{i}-f_{i}(x)\right] /\left(\bar{f}_{i}-\underline{f}_{i}\right)\right|^{p}\right\}^{1 / p} \quad \forall x \in X,
$$

where $w_{i}$ is a weight, chosen by the decision maker, which measures the relative importance of the $i$ th criterion and

$$
\underline{f}_{i}=\min _{x \in X} f_{i}(x) .
$$

Thus, the measure of closeness is given by the $L_{p}$-norm of the vector whose $i$ th component is

$$
w_{i} /\left[\bar{f}_{i}-f_{i}(x)\right] /\left(\bar{f}_{i}-\underline{f}_{i}\right), \quad i=1, \ldots, m .
$$

A gain, if every $f_{i}$ is $\mathrm{DC}$, with a $\mathrm{DC}$ decomposition known, finding the best compromise solution amounts to solving a $D C$ problem with a $D C$ representation of the objective known. 
Compromise programming is not the only M CDM approach in which Proposition 1.1 can be applied, since (as it is shown in R ef. 19) any of the goal programming, multiobjective programming, and compromise programming approaches can be derived from the following distance function model:

$$
\min _{x \in X}\left\{\sum_{i=1}^{m} w_{i}^{p}\left|\left[b_{i}-f_{i}(x)\right] / k_{i}\right|^{p}\right\}^{1 / p},
$$

where $b_{i}$ is the aspiration level for the $i$ th criterion and $k_{i}$ a normalizing constant. If every $f_{i}$ is $\mathrm{DC}$, and if a $\mathrm{DC}$ representation is known for all of them, Proposition 1.1 will provide a DC decomposition for the objective function of (13).

H ence, standard branch-and-bound algorithms can be used to solve it, whereas the techniques proposed so far perform only local searches (R efs. 20-21), so they can be trapped in locally (not globally) optimal decisions, as illustrated below.

Example 2.2. Following (13), we have considered a goal-programming problem with $m=5, w_{i}=k_{i}=1, i=1, \ldots, m$, and functions and goals given by

$$
\begin{array}{ll}
f_{1}(x, y)=x+10 \cos (5 \pi y), & b_{1}=10, \\
f_{2}(x, y)=x^{2}-6 x+y^{2}, & b_{2}=0, \\
f_{3}(x, y)=10 x+7 y, & b_{3}=70, \\
f_{4}(x, y)=x^{2}-6 x+4 y, & b_{4}=11, \\
f_{5}(x, y)=x^{2}+y^{2}, & b_{5}=9 .
\end{array}
$$

The feasible region is the rectangle

$$
S=\left\{(x, y) \in \mathbb{R}^{2}: 0 \leq x \leq 6,0 \leq y \leq 4\right\} .
$$

Figure 3 contains a plot of the objective function for $p=2$, showing its multimodal character.

The problem above has been solved for a grid of 50 values of $p$ between 1 and 2, yielding the optimal solutions $x_{j}^{*}, j=1, \ldots, 50$, depicted in Fig. 4. The trajectory traced by these points seems not to be continuous, due to changes in the local minimum providing the global optimum. 


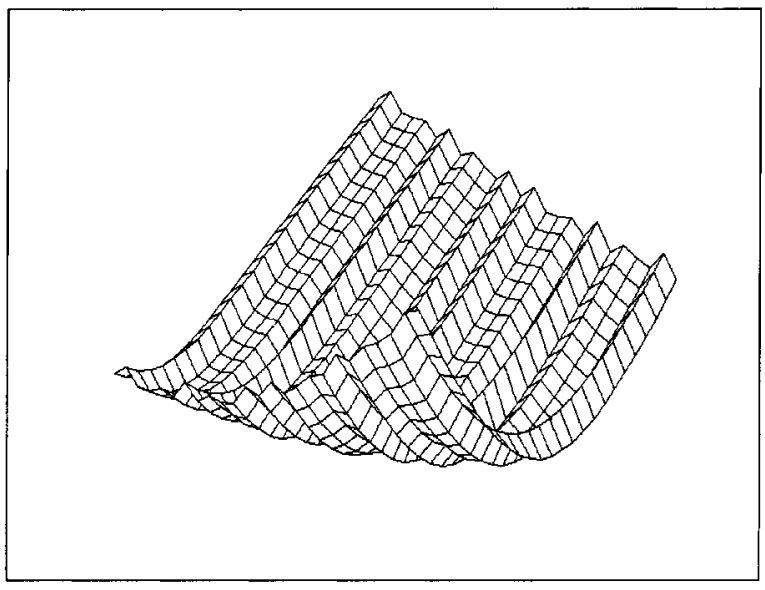

Fig. 3. Objective function, Example 2.2.

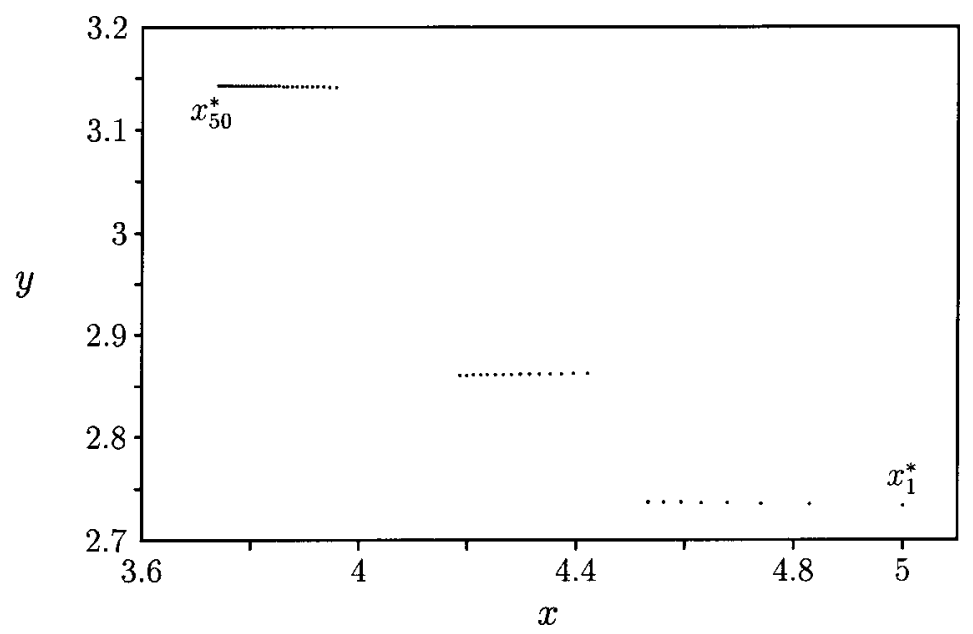

Fig. 4. Trajectories of optimal solutions.

\section{References}

1. Horst, R., and ThoA , N. V., DC Programming: Overview, J ournal of Optimization Theory and A pplications, Vol. 103, pp. 1-43, 1999.

2. Horst, R., and TUY, H., Global Optimization: Deterministic Approaches, Springer Verlag, Berlin, G ermany, 1996.

3. TuY, H., DC Optimization: Theory, Methods, and Algorithms, $\mathrm{H}$ andbook of G lobal Optimization, E dited by R. H orst and P. M. Pardalos, K luwer A cademic Publishers, Dordrecht, Holland, pp. 149-216, 1995. 
4. Hartman, P., On Functions Representable as a Difference of Convex Functions, Pacific Journal of M athematics, Vol. 9, pp. 707-713, 1959.

5. TuY, H., Convex Analysis and Global Optimization, Kluwer Academic Publishers, Dordrecht, Holland, 1998.

6. MICHEL Ot, C., The Mathematics of Continuous Location, Studies in L ocational A nalysis, Vol. 5, pp. 59-83, 1993.

7. Rock afellar, R. T., Convex Analysis, Princeton U niversity Press, Princeton, N ew J ersey, 1970.

8. A NeJA, Y. P., and PARLAR, M., Algorithms for Weber Facility Location in the Presence of Forbidden Regions and/or Barriers to Travel, Transportation Science, Vol. 28, pp. 70-76, 1994.

9. Brimberg, J., and Wesolowsky, G. O., The Rectilinear Distance Minimum Problem with Minimum Distance Constraints, Location Science, Vol. 3, pp. 203215, 1995.

10. Hamacher, H. W., and N ICkel, S., Restricted Planar Location Problems and Applications, N aval R esearch Logistics, Vol. 42, pp. 967-992, 1995.

11. Bittner, L., Some Representation Theorems for Functions and Sets and Their Application to Nonlinear Programming, Numerische M athematik, Vol. 16, pp. 32-51, 1970.

12. Hiriart-U r Ruty, J. B., Generalized Differentiability, Duality, and Optimization for Problems Dealing with Differences of Convex Functions, Convexity and Duality in Optimization, Edited by J. Ponstein, Springer Verlag, Berlin, Germany, pp. 37-69, 1985.

13. Blanquero, R., and Carrizosa, E., On Covering Methods for DC Optimization, To appear in J ournal of G lobal Optimization.

14. Hiriart-Urruty. J. B., Convex Analysis and Minimization Algorithms, I, Springer Verlag, Berlin, G ermany, 1993.

15. Bazaraa, M. S., Sherali, H. D., and Shetty, C. M., Nonlinear Programming: Theory and Algorithms, J ohn W iley and Sons, N ew Y ork, NY, 1993.

16. Zeleny, M., Compromise Programming, M ultiple-Criteria Decision M aking, Edited by J. L. Cochrane, and M. Zeleny, U niversity of South Carolina Press, Columbia, South Carolina, pp. 262-301, 1973.

17. Zeleny, M., A Concept of Compromise Solutions and the Method of the Displaced Ideal, Computers and Operations R esearch, Vol. 1, pp. 479-496, 1974.

18. Zeleny, M., Multiple-Criteria Decision Making, Springer Verlag, Berlin, Germany, 1976.

19. Romero, C., Handbook of Critical Issues in Goal Programming, Pergamon Press, Oxford, England, 1991.

20. Saber, H. M., and Ravindran, A., Nonlinear Goal Programming Theory and Practice: A Survey, Computers and Operations R esearch, Vol. 20, pp. 275-291, 1993.

21. Saber, H. M., and Ravindran, A., A Partitioning Gradient Based (PGB) Algorithm for Solving Nonlinear Goal Programming Problems, Computers and Operations R esearch, Vol. 23, pp. 141-152, 1995. 